\title{
Hydrogen and Methoxy Coadsorption in the Computation of the Catalytic Conversion of Methanol on the Ceria (111) Surface
}

\author{
Ariana Beste ${ }^{\mathrm{a}, \mathrm{b}, *}$, Steven H. Overbury ${ }^{\mathrm{c}}$ \\ ${ }^{a}$ Joint Institute for Computational Sciences, The University of Tennessee, Oak Ridge, \\ TN 37831 \\ ${ }^{b}$ Center for Nanophase Materials Sciences, Oak Ridge National Laboratory, Oak Ridge, \\ TN 37831 \\ ${ }^{c}$ Chemical Sciences Division, Oak Ridge National Laboratory, Oak Ridge, TN 37831
}

\begin{abstract}
Methanol decomposition to formaldehyde catalyzed by the ceria (111) surface has been investigated using the DFT $+U$ method. Our results rationalize experimental temperature programmed desorption experiments on the fully oxidized surface. Particular attention has been paid to the model correctness of methoxy with coadsorbed hydrogen on the surface. This issue has been raised by the experimental observation of water desorption at low temperature removing hydrogen from the system. Our investigation also includes hydrogen diffusion as a means of hydrogen removal. We show that the $\mathrm{CH}_{3} \mathrm{O}^{-} / \mathrm{H}^{+}$ion pair remains at large separation, rendering the coadsorbed model as appropriate in contrast to a neutral isolated methoxy, for which the dehydrogenation barrier becomes negligible. We find that the presence of methoxy reduces the reaction energy for water formation considerably. In
\end{abstract}

\footnotetext{
* Corresponding author

Email address: bestea@ornl.gov (Ariana Beste)
} 
addition, the preference of the electron to locate at the methoxy oxygen results in a dehydrated surface that does not contain $\mathrm{Ce}^{3}+$ ions, despite the existence of a vacancy.

Keywords: methoxy, formaldehyde, DFT $+\mathrm{U}$, transition state, dehydrogenation, hydrogen diffusion, water formation

This manuscript has been authored by UT-Battelle, LLC under Contract No. DE-AC05-000R22725 with the U.S. Department of Energy. The United States Government retains and the publisher, by accepting the article for publication, acknowledges that the United States Government retains a non-exclusive, paid-up, irrevocable, world-wide license to publish or reproduce the published form of this manuscript, or allow others to do so, for United States Government purposes. The Department of Energy will provide public access to these results of federally sponsored research in accordance with the DOE Public Access Plan. 


\section{Introduction}

Cerium oxide is used as a catalyst and as a support material for a variety of catalytic processes $[1,2]$. It facilitates acid-base [3] as well as redox reactions giving the opportunity to tune the catalyst cooperatively. It also shows an excellent oxygen-storage capability, which is exploited when used as support for platinum group metals in three-way catalysts to reduce the tailpipe emissions of hydrocarbons, carbon monoxide, and nitrogen oxides [4]. In addition, ceria enhances the catalytic activity of transition metals, for instance, in the water-gas-shift reaction [5] and steam reforming of hydrocarbons [2]. When ceria is used to manufacture electrodes for solid oxide fuel cells [1], its redox properties are the decisive characteristic. Recent developments in the controlled synthesis of nanostructured ceria [6] give rise to applications that yet have to be fully explored. Here, the sensitivity of chemical selectivity towards surface structure and nanoshape morphology are most intriguing $[7]$.

In order to obtain insights into the structure dependence in chemical reactivity, we and others have explored surface chemistry on oriented single crystal films of $\mathrm{CeO}_{2}[8,9]$ and catalytic reactions on shape-controlled $\mathrm{CeO} 2$ nanoparticles $[7,10,11]$. Guided by such experimental studies on structurally well-defined surfaces it is of complimentary value to use computational methods to probe reaction pathways and surface molecular interaction $[12,13]$. An excellent review on computational work on ceria surface chemistry has been published [14]. Although cerium in its reduced form is difficult to de-

scribe by density functional theory (DFT) due to the self-interaction error present in approximate functionals, DFT has become a standard tool to in- 
vestigate surface reactions over ceria. In particular, the $\mathrm{DFT}+\mathrm{U}$ method is often applied, which adds on-site correlation to partially correct the error and to localize atomic-like $4 \mathrm{f}$ electrons.

In this work, we study methanol conversion on the ceria (111) surface. DFT calculations on the dissociative adsorption of methanol on the (111) surface $[15,12]$ and kinetic data for initial bond-breaking on the (110) surface [16] are available. Detailed investigations of the methanol decomposition mechanism on the ceria (111) surface up to formaldehyde [17] and complete oxidation to $\mathrm{CO}$ and $\mathrm{H}_{2}$ [18] have been undertaken very recently. In both studies the DFT $+\mathrm{U}$ method has been employed while, in addition, a comparison with a presumably more reliable hybrid DFT functional is provided in reference [17]. Other relevant work includes the investigation of ceria support effects on methanol oxidation [19] and DFT studies on formaldehyde adsorption [20, 21] and oxidation [21] on ceria. Pathways for dehydrogenation and dehydration catalyzed by ceria surfaces for methanol's larger homolog, ethanol, were computationally examined as well [13].

Dehydrogenation of alcohols is a probe reaction to characterize catalytic properties of oxide materials $[22,23]$. Methanol, the simplest of these alcohols, has been examined experimentally with different techniques. Specifically, temperature programmed desorption (TPD) experiments [24, 25, 26] have shown a product distribution on the oxidized surface that corresponds to a seemingly simple reaction mechanism; methanol, water, and formaldehyde were detected. Since methoxy has been found as an intermediate on the ceria surface [24], formaldehyde formation only entails three reaction steps: methanol adsorption, $\mathrm{O}-\mathrm{H}$ cleavage, and finally $\mathrm{C}-\mathrm{H}$ bond cleavage. 
However, low-temperature water desorption is puzzling since multiple studies $[27,28,29,30]$ have reported reaction energies above $2 \mathrm{eV}$ for water formation. It also potentially complicates the mechanism since implied vacancy creation may alter the decomposition chemistry even on the initially fully oxidized surface. Another consequence of water desorption is that methoxy loses its coadsorbed hydrogen. The typical computational approach is to model methoxy dehydrogenation in the presence of its coadsorbed hydrogen $[17,18]$. However, this might not be correct if hydrogen is consumed through water formation.

The effect of hydrogen and methoxy coadsorption on various aspects of methanol decomposition to formaldehyde on the ceria surface, including hydrogen diffusion, is the main focus of this work. In the following we report results for methanol oxidation to formaldehyde catalyzed by the ceria (111) surface employing the commonly used model, in which hydrogen is coadsorbed. We then discuss hydrogen diffusion with and without coadsorbed methoxy, the nature of the hydrogen-methoxy interaction, carbon/hydrogen bond cleavage in the absence of coadsorbed hydrogen, and water formation in the presence of methoxy. We show that the hydrogen-methoxy interaction remains ionic even at large separation.

\section{Computational Details}

All electronic structure calculations were carried out using the projectoraugmented wave (PAW) method $[31,32]$ as implemented in the Vienna ab initio simulation package (VASP) $[33,34,35,36]$. Although we mostly investigated methanol conversion on the fully oxidized surface, adsorbate oxidation 
generally leads to surface reduction. We, therefore, employed the $\mathrm{PBE}+\mathrm{U}$ $[37,38]$ functional within the DFT formalism to correct for shortcomings of the standard gradient-corrected exchange-correlation functional (PBE) to describe the reduced surface. While a value of $5 \mathrm{eV}$ for $\mathrm{U}$ is optimized to position occupied Ce 4 f states about $1.3 \mathrm{eV}$ above the oxygen $2 \mathrm{p}$ states in bulk $\mathrm{Ce}_{2} \mathrm{O}_{3}$ [30] and is often used in calculations of reduced ceria [14], a value of 2$3 \mathrm{eV}$ for $\mathrm{U}$ was suggested for the description of the redox chemistry over ceria [39] and bulk reduction energies [40] and a value of $2 \mathrm{eV}$ was recommended for activation barriers [41]. A minimum value of $\mathrm{U}=3.0 \mathrm{eV}$ was determined for the f-electrons to localize [42]. Here, we chose a value of $3.0 \mathrm{eV}$ for the effective $\mathrm{U}$ parameter. This resulted in a lattice constant for $\mathrm{CeO}_{2}$ of 5.46 $\AA$, intermediate between the lattice constants obtained with $\mathrm{PBE}(\mathrm{U}=0)$ and $\mathrm{PBE}+\mathrm{U}(\mathrm{U}=4.5)[43]$ and compared to the experimental value of $5.41 \AA$.

We employed spin-polarized functionals, an energy cut off of $700 \mathrm{eV}$, and dipole corrections perpendicular to the surface. Dispersion corrections were included through the D3 method by Grimme [44]. The surface cell was constructed by stacking three units of oxygen-cerium-oxygen layers for a total of 9 atomic layers within the slab and a vacuum layer of $15 \AA$. For the majority of the calculations we used a $\mathrm{p}(2 \times 2)$ expansion of the surface cell, which corresponds to about $1 / 4 \mathrm{ML}$ coverage or $1.9 \mathrm{~nm}^{-2}$. We employed a $\Gamma$-centered 3-3-1 Monkhorst-Pack k-point mesh for the $\mathrm{p}(2 \times 2)$ cell, which we found converged within $0.01 \mathrm{eV}$ for the dissociative adsorption energy of methanol compared to the 4-4-1 k-point mesh. In order to investigate the distance dependence of the interaction between methoxy and coadsorbed hydrogen, we increased the size of the surface cell to $\mathrm{p}(8 \mathrm{x} 8)$. For the latter, 
we performed $\Gamma$-point calculations.

The top 6 atomic layers were relaxed during minimization and reaction path optimization, while the bottom 3 layers were fixed. Minimum energy paths were preoptimized with the nudged elastic band method [45] (NEB) and transition states were located with the climbing image nudged elastic band method [46] (CI-NEB), where we used tools provided by the Henkelman group to set up the input. Stationary points on the potential energy surface were confirmed by frequency analysis. Bader charge analysis was carried out with the code provided by the Henkelman group [47, 48, 49]. All systems were modelled as neutral unless otherwise noted. The dissociative adsorption energy is defined as the sum of adsorption energy and dissociation energy. For the case of methanol, it is the energy difference between methoxy and coadsorbed hydrogen on the surface and the sum of methanol and clean surface energy. Formaldehyde adsorption energies are given within the context of methanol conversion; i.e., with reference to two hydrogen adsorbed on the surface.

\section{Results and Discussion}

Methanol adsorbs on the (111) ceria surface in an atop position over cerium, see Figure $1 \mathrm{a}$ ), with an adsorption energy of $0.81 \mathrm{eV}$. This is in agreement with previous work using slightly different methods $[15,12]$. Notice the tilt in the $\mathrm{C}-\mathrm{O}$ axis maximizing orbital overlap between the oxygen electron pair and the orbitals of the cerium atom. Methanol O-H cleavage is close to thermoneutral and proceeds through a small barrier of $0.09 \mathrm{eV}$. The corresponding transition state and the $\mathrm{O}-\mathrm{H}$ cleavage product are shown 


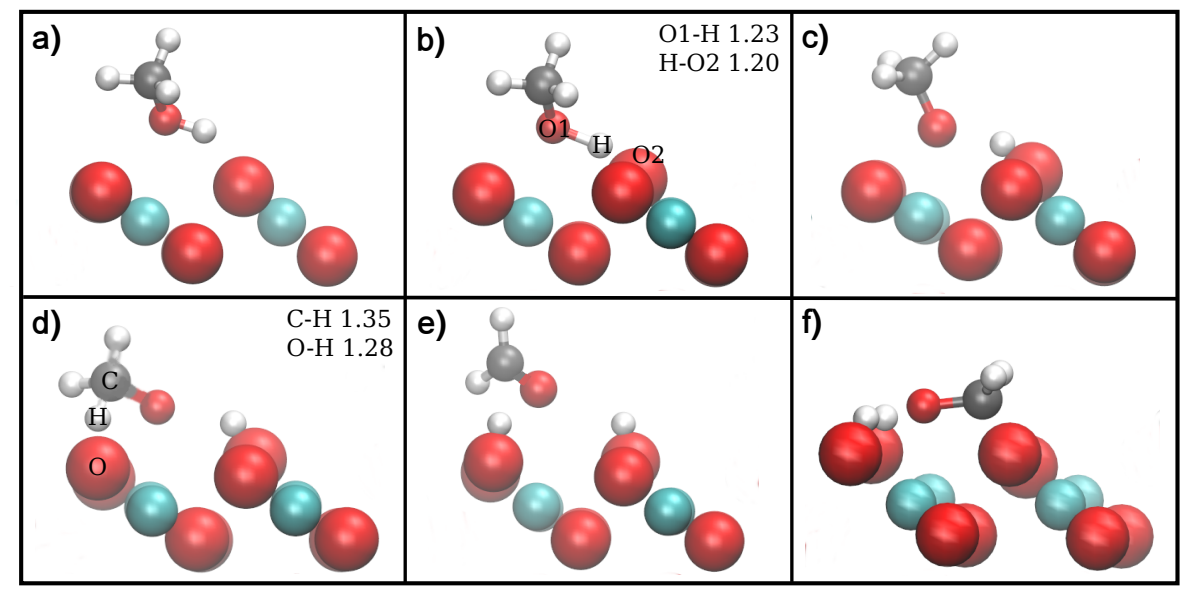

Figure 1: Side view of lowest energy structures for a) methanol, c) methoxy, e) physisorbed formaldehyde, and f) chemisorbed formaldehyde with coadsorbed hydrogen atoms, and of transition states for b) methanol $\mathrm{O}-\mathrm{H}$ cleavage and d) methoxy $\mathrm{C}-\mathrm{H}$ cleavage on the oxidized (111) ceria surface; distances in $\AA$; atoms colored as follows, white: hydrogen, gray: carbon, red: oxygen, cyan: cerium.

in Figure $1 \mathrm{~b}$ ) and c), respectively. The $\mathrm{O}-\mathrm{H}$ cleavage pathway is given in the Supporting Information, Figure 1. The geometric arrangement in Figure $1 \mathrm{~b}$ ) suggests that there is an attractive force between methoxy and the coadsorbed hydrogen. Methoxy reacts further via $\mathrm{C}-\mathrm{H}$ bond cleavage to yield formaldehyde that is physisorbed to the surface with an adsorption energy of $0.40 \mathrm{eV}$ and depicted in Figure $1 \mathrm{e}$ ). This requires an activation energy of $1.29 \mathrm{eV}$; see Figure $1 \mathrm{~d}$ ) for the transition state and the Supporting Information, Figure 2 for the reaction path. Although frequency analysis reveals no imaginary frequencies for physisorbed formaldehyde in Figure $1 \mathrm{~d}$ ), we do not detect a barrier for conversion of the physisorbed species to the chemisorbed structure shown in Figure $1 \mathrm{f}$ ). The calculated NEB path can be found in the Supporting Information, Figure 3. Chemisorbed formaldehyde has an 
adsorption energy of $1.45 \mathrm{eV}$ and is characterized by a strong interaction between the carbon atom and a surface oxygen leading to its description as dioxymethylene [50]. The rehybridized tetrahedral bond pattern in carbon indicates that the surface species is best described as a bi-dentate formaldehyde adsorbate $[20,21]$. Our calculations show that the chemisorbed species is formed with the physisorbed species as intermediate; i.e., we did not find a reaction path from methoxy directly to the chemisorbed species (see Supporting Information, Figure 4). The reaction profile for the conversion of methanol to formaldehyde on the oxidized (111) ceria surface is given in Figure 2 and compares well with other recent work [17, 18]. The largest discrepancy can be found in the stability of physisorbed and chemisorbed formaldehyde on the surface. While we calculate methoxy $\mathrm{C}-\mathrm{H}$ cleavage to the physisorbed species to be close to thermoneutral, it was reported to be exothermic with $-0.76 \mathrm{eV}$ [17]. Also the chemisorbed formaldehyde appears to be less stable in our calculations compared to other work [18] by about $0.5 \mathrm{eV}$. In addition, we compute a higher barrier for $\mathrm{C}-\mathrm{H}$ cleavage by $0.26 \mathrm{eV}$ than in reference [18]. Comparison of the PBE+U with HSE results (including dispersion corrections) [17] shows that the $\mathrm{PBE}+\mathrm{U}$ method overstabilizes the reduced Ce state resulting in larger adsorption energies for formaldehyde and lower $\mathrm{C}-\mathrm{H}$ cleavage barriers compared to results of the hybrid functional. Note that an effective $\mathrm{U}$ of $4.5 \mathrm{eV}$ was used in those studies [18, 17] while we chose $3 \mathrm{eV}$ for the $\mathrm{U}$ parameter, which seems to agree better with HSE values.

The desorption peak observed in TPD experiments on the oxidized surface at $570 \mathrm{~K}$ assigned to formaldehyde [24] implies a desorption energy of 


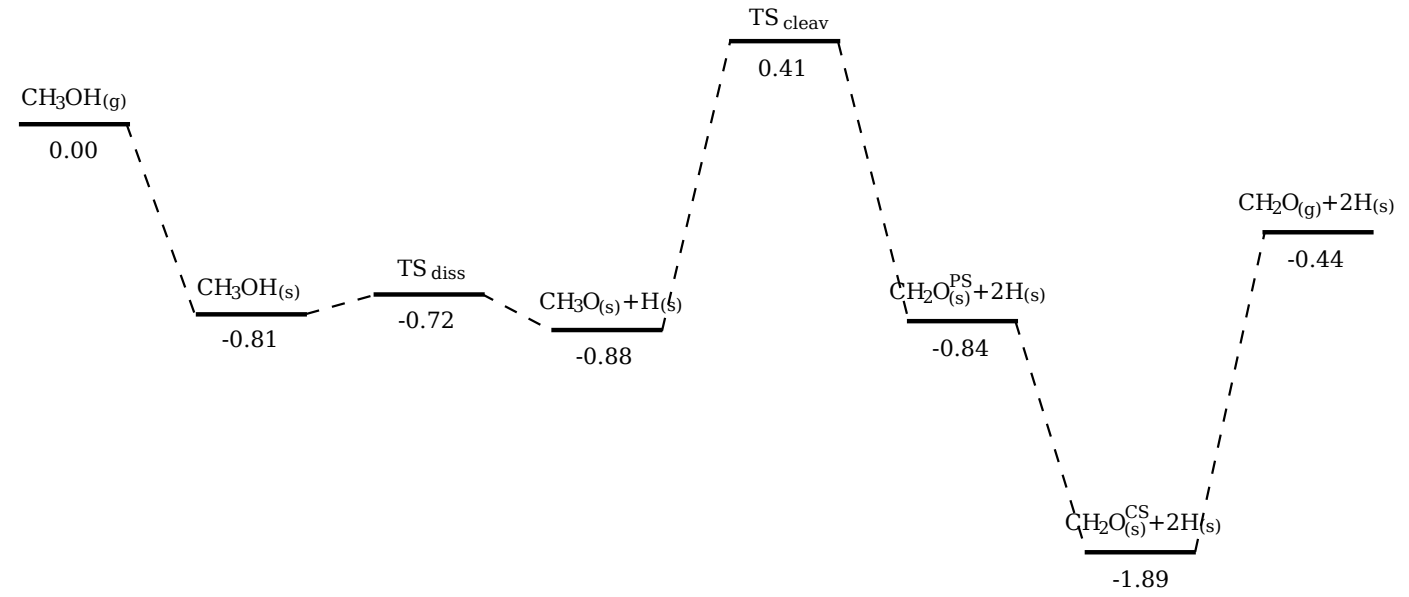

Figure 2: Reaction profile for methanol conversion to formaldehyde on the oxidized (111) ceria surface; energies in eV, subscript (g) indicates gas phase, subscript (s) indicates adsorbed on surface, superscript PS stands for physisorbed and superscript CS for chemisorbed, $\mathrm{TS}_{\text {diss }}$ is the transition state for methanol $\mathrm{O}-\mathrm{H}$ cleavage and $\mathrm{TS}_{\text {cleav }}$ is the transition state for methoxy C-H cleavage.

$1.52 \mathrm{eV}$ using Redhead analysis [51]. This is consistent with our calculated data assuming that the peak corresponds to the desorption of chemisorbed formaldehyde that forms (immediately) without barrier from the physisorbed species, which, in turn, is the product of $\mathrm{C}-\mathrm{H}$ cleavage in methoxy requiring an activation energy below that value. Another prominent feature of the TPD spectrum is the water peak at around $200 \mathrm{~K}$. The hydrogen in the desorbed water was shown to originate from the alcohol group in methanol, which indicates that methanol $\mathrm{O}-\mathrm{H}$ cleavage occurs with an activation energy smaller than $0.53 \mathrm{eV}$ according to Redhead analysis. A small barrier for methanol O-H cleavage is confirmed by our calculation. It also raises the question if hydrogen should be modelled as a coadsorbate as commonly done $[17,18]$ including the current work. This question will be addressed below. 
Table 1: Energies for hydrogen diffusion pathways with and without coadsorbed metoxy; path 1 and path 3 proceed through an intermediate: $\Delta \mathrm{E}$ is the energy difference between intermediate and reactant, path 2 proceeds through a transition state: $\Delta \mathrm{E}$ is the activation energy; path 1) - diffusion above surface, path 2) - diffusion within top layer, path 3) diffusion below surface.

\begin{tabular}{l|l|l}
\multirow{2}{*}{} & \multicolumn{2}{|c}{$\Delta \mathrm{E}[\mathrm{eV}]$} \\
\cline { 2 - 3 } & no & yes \\
\hline path 1) & 3.15 & \\
path 2) & 1.69 & 2.22 \\
path 3) & 1.54 & 1.87
\end{tabular}

Since hydrogen diffusion may result in the translation of hydrogen away from the dehydrogenation site as well, we first investigate hydrogen diffusion with and without coadsorbed methoxy.

Although more pathways for hydrogen diffusion are conceivable, in the following we discuss three pathways where hydrogen diffuses 1) above the surface, 2) within the top oxygen layer, and 3) below the surface. A summary of the required energies is given in Table 1. Due to its small size, hydrogen can be incorporated into the lattice forming high energy structures as, for instance, shown in Figure 3 a). This (example) structure is $1.03 \mathrm{eV}$ higher in energy than the energetically preferred structure Figure $3 \mathrm{~b}$ ), where hydrogen is adsorbed on the surface. Diffusion of hydrogen to a neighbouring surface oxygen above the surface (path 1) proceeds through an intermediate depicted in Figure $3 \mathrm{c}$ ), where the hydrogen atom is located above a third layer oxygen. This is a high-energy pathway where the intermediate is $3.15 \mathrm{eV}$ higher in 


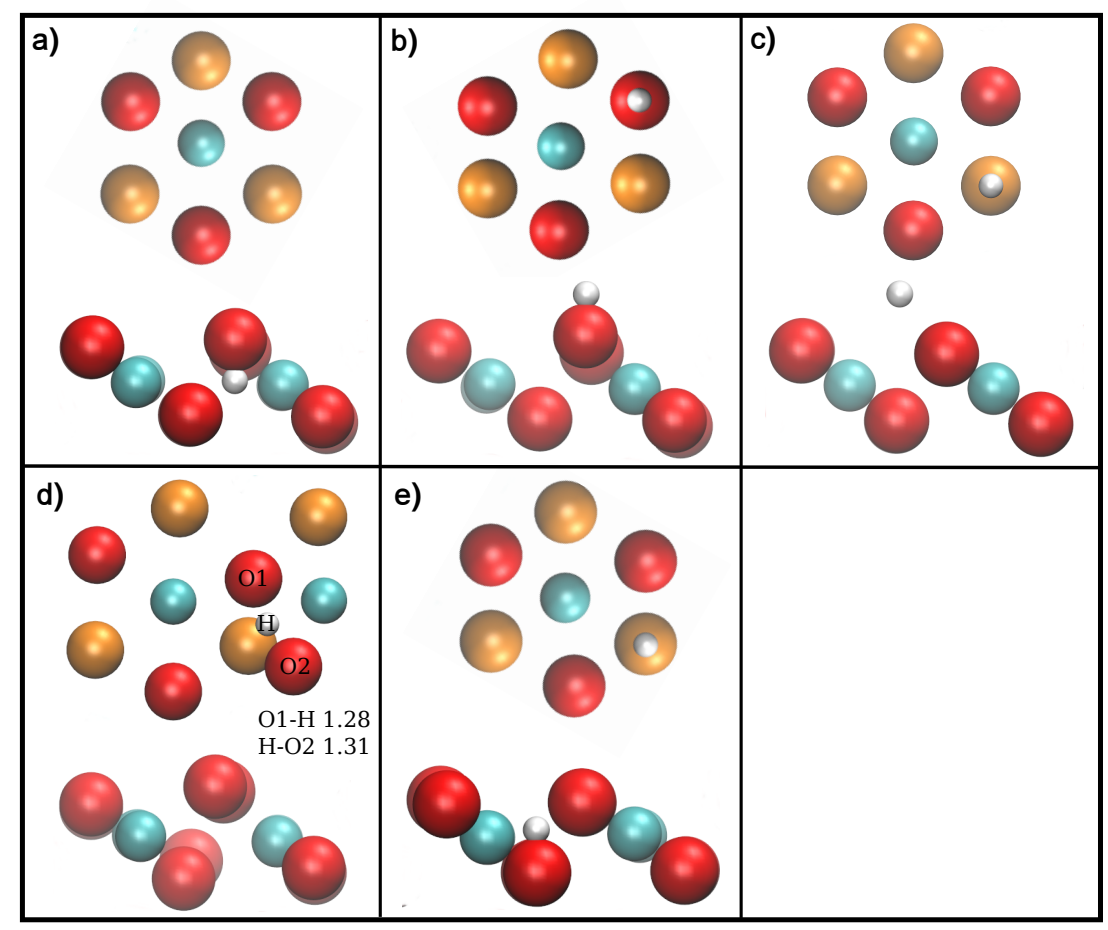

Figure 3: Top (upper) and side (lower) view of a) hydrogen lattice incorporation, b) surface adsorbed hydrogen, c) intermediate for hydrogen diffusion above the surface (path 1), d) transition state for hydrogen diffusion within top layer (path 2), and e) intermediate for hydrogen diffusion below the surface (path 3 ); distances in $\AA$; atoms colored as follows, white: hydrogen, cyan: cerium, red: oxygen, orange: oxygen in second layer in top view.

energy than the lowest energy structure. While one of the cerium atoms in is initially reduced, a distinctive feature of the intermediate is that there is a nonzero magnetization on hydrogen and no magnetization on cerium. This suggests that the hydrogen in the intermediate is atomic and not bound to the surface. We do not find a barrier for intermediate formation along path 1), which is shown in the Supporting Information, Figure 5. Another possible diffusion path for hydrogen from a surface oxygen to its neighbour 
is within the top layer. Path 2) has a transition state given in Figure 3 d) that is characterized by an oxygen-hydrogen-oxygen bridge. The surface remains reduced, hydrogen bound to oxygen, and there is no magnetization on hydrogen throughout the path. This results in a lower energy path than path 1) with an activation barrier of $1.69 \mathrm{eV}$. The CI-NEB path can also be found in the Supporting Information, Figure 6. A path with similar energetic requirement is obtained when hydrogen diffuses into the surface (path 3). An intermediate is formed that is $1.54 \mathrm{eV}$ higher in energy than the initial structure and displayed in Figure 3 e). This intermediate has been identified in prior work $[28,52]$. Similar to path 2) and in contrast to the high energy path 1), hydrogen has zero magnetic moment along path 3) while one cerium atom in the surface is reduced to $\mathrm{Ce}^{3+}$. The NEB path to the intermediate is endergonic and does not reveal a transition state (as given in the Supporting Information, Figure 7). Note that due to cell size limitations, we choose the $\mathrm{Ce}^{3+}$ to be located next to the hydrogen carrying oxygen in the initial structures for path 2) and 3) while an energetically lower structure (by 0.1 $\mathrm{eV}$ ) is obtained when the $\mathrm{Ce}^{3+}$ is positioned next neighbour to that oxygen. The latter is the initial structure for path 1 ).

Next, we consider the effect of coadsorbed methoxy on the hydrogen diffusion pathways. We did not find a high energy pathway equivalent to path 1). When methoxy is coadsorbed, the intermediate of path 1) converts during optimization into methanol adsorbed on the surface. The NEB path 2) with coadsorbed methoxy is included in the Supporting Information, Figure 8. Again, we find a transition state where an oxygen-hydrogen-oxygen bridge is formed within the top surface layer and which is displayed in Figure 4 a). The 


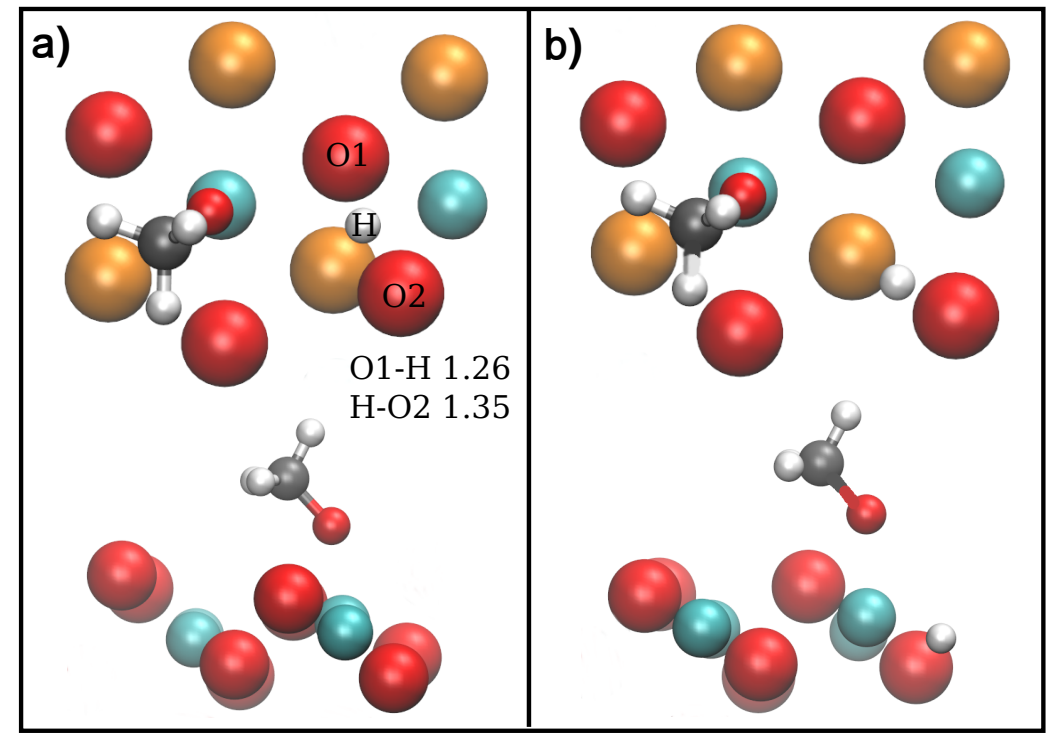

Figure 4: Top (upper) and side (lower) view of a) transition state for hydrogen diffusion within top layer (path 2), and b) intermediate for hydrogen diffusion below the surface with coadsorbed methoxy (path 3); distances in $\AA$; atoms colored as follows, white: hydrogen, cyan: cerium, red: oxygen, orange: oxygen in second layer in top view.

presence of methoxy increases the activation barrier to $2.22 \mathrm{eV}$. Path 3) with coadsorbed methoxy proceeds through an intermediate where the hydrogen is displaced from the on-top position above the third-layer oxygen and is shown in Figure $4 \mathrm{~b}$ ). The diffusion path is given in the Supporting Information, Figure 9. Interestingly, the plateau in the reaction path for the conversion to the intermediate corresponds to minimum structure Figure 3 a) with coadsorbed methoxy. We also find for path 3) that coadsorption of methoxy raises the path energy to $1.87 \mathrm{eV}$. From our calculations, it appears that the energy needed for hydrogen diffusion with and without coadsorbed methoxy is too high to contribute to the mechanism of water formation around $200 \mathrm{~K}$ in the TPD spectrum on the oxidized ceria surface. 
While C-H cleavage in alkoxy intermediates occurs concurrently with surface reduction [13], the surface remains fully oxidized when methoxy and hydrogen are coadsorbed. Consequently, hydrogen diffusion as modelled above without coadsorbed methoxy is representative for a hydrogen formed through, for instance, C-H cleavage but might not be for a hydrogen formed through methanol O-H cleavage. To analyse the methoxy/hydrogen interaction further, we compute Bader charges and monitor the magnetic moments for neutral and charged systems. Charged systems are modelled by adding or removing one electron from the calculations. Bader charges and magnetic moments can be found in Table 2. We observe that hydrogen $\left(\mathrm{H}_{(S)}\right.$ in Table 2) donates its electron, which is accepted by a cerium atom when methoxy is not coadsorbed. If that electron is taken out of the system $\left(\left[\mathrm{H}_{(S)}\right]^{+}\right.$in Table 2), the hydrogen atom remains largely positive and all cerium atoms are in an oxidized form. In contrast, methoxy on the surface $\left(\mathrm{CH}_{3} \mathrm{O}_{(\mathrm{S})}\right.$ in Table 2) retains unpaired electron density on the methoxy oxygen atom. When an additional electron is added $\left(\left[\mathrm{CH}_{3} \mathrm{O}_{(\mathrm{S})}\right]^{-}\right.$in Table 2$)$, no magnetic moments are detected with a large negative charge on methoxy, suggesting that an electron pair has been formed yielding negatively charged methoxy on the surface. Comparing charges and magnetic moments of $\left[\mathrm{H}_{(S)}\right]^{+}$and $\left[\mathrm{CH}_{3} \mathrm{O}_{(\mathrm{S})}\right]^{-}$in Table 2 to methoxy with coadsorbed hydrogen indicates that methoxy and hydrogen are best described as an ion pair $\left(\mathrm{CH}_{3} \mathrm{O}^{-} / \mathrm{H}^{+}\right)$. For an oppositely charged ion pair, we expect an attractive interaction that is strongest at short distance. It is possible that for separated ions, a different electronic solution becomes energetically lower; for instance, a radical solution as for isolated methoxy or hydrogen on the surface. The Bader charges 
of methoxy and coadsorbed hydrogen (given in the Supporting Information) are largely independent of the distance between them, although we record slightly larger charges for the shortest distance. This suggests that the ionic state remains stable, even at large separation. The Coulombic character of the methoxy/hydrogen interaction is demonstrated in Figure 5, where we show dissociative adsorption energies as a function of adsorbate distance. The adsorption energy decreases as a function of distance approaching a value of about $0.5 \mathrm{eV}$, which is a consequence of the decreasing Coulomb stabilization with larger ionic separation. Also included in Figure 5 is the Coulomb energy as a function of distance for two point charges of 0.25 au offset by $0.46 \mathrm{eV}$, which appears to model the decrease in dissociative adsorption energy up to its asymptotic value very well.

The electronic description of methoxy either as radical or as anion with coadsorbed hydrogen make a striking difference as is demonstrated by considering the $\mathrm{C}-\mathrm{H}$ cleavage in methoxy without coadsorbed hydrogen. As discussed above, $\mathrm{C}-\mathrm{H}$ cleavage in methoxy/H has a significant barrier of 1.29 $\mathrm{eV}$. If hydrogen is omitted, $\mathrm{C}-\mathrm{H}$ cleavage in methoxy, which now resembles a radical adsorbed on the surface, proceeds without activation barrier. The corresponding reaction path is given in the Supporting Information, Figure 10.

Another significant effect of methoxy/hydrogen coadsorption is the reduction of vacancy/water formation energy when methoxy is present. Water formation on the ceria surface with simultaneous vacancy creation has been reported to be strongly endothermic by $2.12-2.44 \mathrm{eV}[27,28,29,30]$. This is in contrast to the experimental observation that following low tempera- 
Table 2: Sum of Bader charges of the atoms in methoxy $\left(\mathrm{CH}_{3} \mathrm{O}\right)$ and Bader charges of coadsorbed hydrogen $(\mathrm{H})$ if present (otherwise noted by -) in au and magnetic moments of methoxy oxygen $\left[\mathrm{O}\left(\mathrm{CH}_{3} \mathrm{O}\right)\right]$, coadsorbed hydrogen $(\mathrm{H})$ if present, and the sum of magnetic moments of cerium atoms $(\mathrm{Ce})$ in $\mu_{B}$ for neutral: methoxy with coadsorbed hydrogen $\mathrm{CH}_{3} \mathrm{O}_{(\mathrm{S})}+\mathrm{H}_{(\mathrm{S})}$, methoxy $\mathrm{CH}_{3} \mathrm{O}_{(\mathrm{S})}$, and hydrogen $\mathrm{H}_{(\mathrm{S})}$; for negatively charged: methoxy $\left[\mathrm{CH}_{3} \mathrm{O}_{(\mathrm{S})}\right]^{-}$; and positively charged systems: hydrogen $\left[\mathrm{H}_{(\mathrm{S})}\right]^{+}$adsorbed on the (111) ceria surface.

\begin{tabular}{c||c|c||c|c|c}
\multirow{2}{*}{ adsorbates } & \multicolumn{2}{|c||}{ charges $[\mathrm{au}]$} & \multicolumn{3}{|c}{ magnetic moments $\left[\mu_{B}\right]$} \\
\cline { 2 - 6 } & $\mathrm{CH}_{3} \mathrm{O}$ & $\mathrm{H}$ & $\mathrm{O}\left(\mathrm{CH}_{3} \mathrm{O}\right)$ & $\mathrm{H}$ & $\mathrm{Ce}$ \\
\hline $\mathrm{CH}_{3} \mathrm{O}_{(\mathrm{S})}+\mathrm{H}_{(\mathrm{S})}$ & -0.58 & 0.65 & 0.00 & 0.00 & 0.00 \\
$\mathrm{CH}_{3} \mathrm{O}_{(\mathrm{S})}$ & -0.19 & - & 0.36 & - & 0.02 \\
{$\left[\mathrm{CH}_{3} \mathrm{O}_{(\mathrm{S})}\right]^{-}$} & -0.66 & - & 0.00 & - & 0.00 \\
$\mathrm{H}_{(\mathrm{S})}$ & - & 0.57 & - & 0.00 & 0.97 \\
{$\left[\mathrm{H}_{(\mathrm{S})}\right]^{+}$} & - & 0.41 & - & 0.00 & 0.00
\end{tabular}

ture methanol desorption water desorbs at around $200 \mathrm{~K}$ without oxygen source other than the surface [24]. Detailed analysis of water formation will be subject of future research. Here, we demonstrate the significance of methoxy coadsorption by calculating the water formation energy for the reaction shown in Figure 6 at 1/2 ML coverage. We do not localize a minimum structure for methoxy that is next neighbor to a vacancy. Instead, during optimization, methoxy migrates into the vacancy where the methoxy oxygen replaces the lattice oxygen that has been incorporated into water. This reduces the water formation energy to $0.79 \mathrm{eV}$. Although somewhat high, this process is thermodynamically attainable at low temperature (around $300 \mathrm{~K}$ according to Redhead analysis). Since the surface in the TPD experiment is 


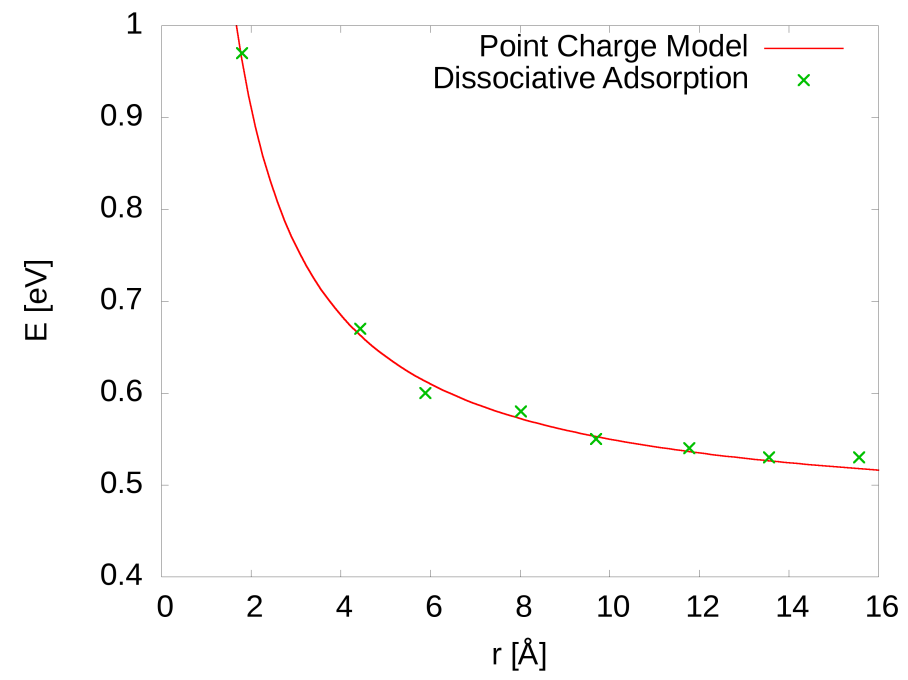

Figure 5: Dissociative adsorption energy of methanol on the (111) ceria surface calculated for a $\mathrm{p}(8 \mathrm{x} 8)$ surface cell as a function of distance $\mathrm{r}$ in $\AA$ between methoxy and coadsorbed hydrogen (x); Coulomb energy in eV for two point charges of 0.25 au offset by $0.46 \mathrm{eV}$ as a function of distance $\mathrm{r}$ in $\AA$ (solid line).

prepared with high methoxy coverage and we demonstrate above that hydrogen (from methanol O-H cleavage) diffusion is unlikely at low temperature, the close proximity of methoxy to the vacancy resulting from water formation is likely. Lowering of the water formation energy is in line with the prior observation that it is easier to create a vacancy on the wet than on the dry ceria surface [27].

Generally, vacancy formation is accompanied by surface reduction since two electrons are left in the surface that occupy empty Ce $4 \mathrm{f}$ sates. However, the magnetic moments of all atoms in the product in Figure 6 are zero. This is not due to the methoxy oxygen residing in the vacancy but due to the preference of the additional electrons to locate at the methoxy oxygen 


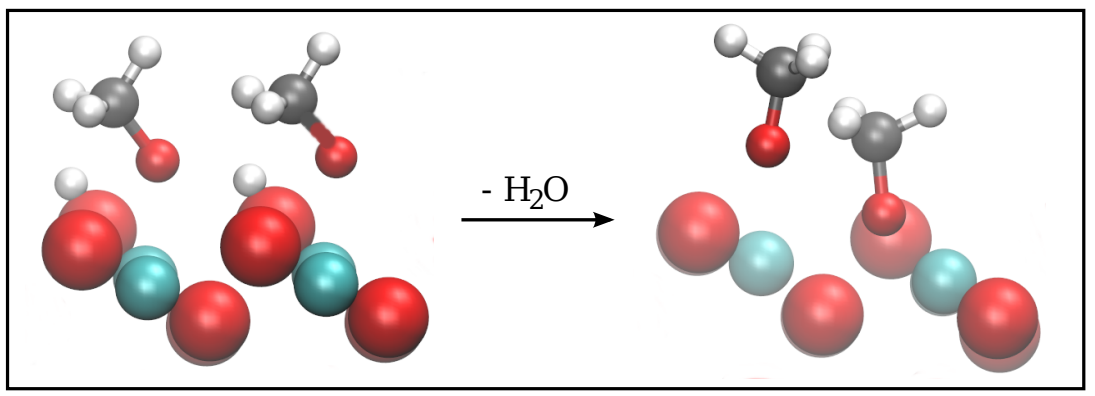

Figure 6: Lowest energy structures relevant to water desorption at 1/2 ML methoxy coverage on the (111) ceria surface; atoms colored as follows, white: hydrogen, gray: carbon, cyan: cerium, red: oxygen.

rather than the cerium $4 \mathrm{f}$ states, much like we demonstrate above for the $\mathrm{CH}_{3} \mathrm{O}^{-} / \mathrm{H}^{+}$ion pair and for the negatively charged system $\left[\mathrm{CH}_{3} \mathrm{O}_{(\mathrm{S})}\right]^{-}$. To show this, we restrict movement of the methoxy oxygen atoms to allow relaxation perpendicular to the surface only during optimization. We then obtain a surface with a vacancy and two neighboring methoxy molecules, which also has zero magnetic moments. This has the important consequence that vacancy formation cannot be equated with surface reduction. It has been pointed out that XPS signals attributed to $\mathrm{Ce}^{3+}$ do not necessarily imply the existence of a vacancy [30]; conversely, the presence of a vacancy is also not always detectable by XPS.

\section{Conclusion}

We have computed the reaction profile for methanol decomposition to formaldehyde on the (111) ceria surface using the DFT $+\mathrm{U}$ method. It appears that the choice of a lower value for the effective $U$ parameter $(\mathrm{U}=3$ $\mathrm{eV}$ ) provides better agreement with hybrid functional results [17] than the 
typically used value of $4.5 \mathrm{eV}[17,18]$. As is common practice $[17,18]$, the dissociated hydrogen remained coadsorbed on the surface. While experimental TPD data [24] for the fully oxidized surface can be rationalized by the computational results, an open question was the correctness of the model including coadsorbed hydrogen because water desorption removing that hydrogen from the surface was observed at low temperature. Since hydrogen diffusion is an alternative route to remove hydrogen from its coadsorbed position, we investigated hydrogen diffusion including the effect of coadsorbed methoxy. We found that methoxy raises the energetic requirements for hydrogen diffusion from about 1.5 to $1.9 \mathrm{eV}$ and that, therefore, hydrogen diffusion has a minor contribution to the mechanism. However, the presence of methoxy lowers the reaction energy of water formation from above $2 \mathrm{eV}$ to about $0.8 \mathrm{eV}$. While kinetic data for this process are forthcoming, the thermodynamic data indicate that the close proximity of methoxy allows water/vacancy formation at low temperature. Still, Bader charge analysis and the dependence of the adsorption energy on the methoxy/hydrogen distance suggest that keeping hydrogen coadsorbed to methoxy is a good model since it ensures the correct ionic state of methoxy in the $\mathrm{CH}_{3} \mathrm{O}^{-} / \mathrm{H}^{+}$ion pair. Even at large methoxy/hydrogen separation we do not observe that the radical state becomes energetically favoured. Neutral methoxy without coadsorbed hydrogen shows radical character, which causes $\mathrm{C}-\mathrm{H}$ cleavage to become barrierless, rendering isolated methoxy an inappropriate model for dehydrogenation. Further, water formation is likely to produce a methoxy located in a vacancy. Therefore, a complete model must include C-H cleavage for methoxy inside a vacancy even on the oxidized surface. An important 
consequence of the preference of electrons to locate on methoxy oxygen over cerium $4 \mathrm{f}$ states is that the existence of a vacancy cannot necessarily be detected by XPS if methoxy is present on the surface. Independently of methoxy being located in the vacancy or not, the methoxy/vacancy system has zero magnetic moments.

\section{Acknowledgements}

This research was sponsored by the Laboratory Directed Research and Development Program of Oak Ridge National Laboratory, managed by UTBattelle, LLC, for the U. S. Department of Energy. This research was in part supported by an allocation of advanced computing resources provided by the National Science Foundation and performed on Darter at the National Institute for Computational Sciences (http://www.nics.tennessee.edu/). This research also used resources of the Oak Ridge Leadership Computing Facility at the Oak Ridge National Laboratory, which is supported by the Office of Science of the U.S. Department of Energy under Contract No. DE-AC0500OR22725.

Supporting Information Available: Supporting information contains NEB and CI-NEB pathways for methanol O-H cleavage, methoxy C-H cleavage to physisorbed and chemisorbed formaldehyde, methoxy $\mathrm{C}-\mathrm{H}$ cleavage without coadsorbed hydrogen, physisorbed to chemisorbed formaldehyde conversion, hydrogen diffusion above the surface, within the top layer, and below the surface, the latter two with and without coadsorbed methoxy, and a table of Bader charges in methoxy and coadsorbed hydrogen as a function of 
methoxy/hydrogen distance. This material is available free of charge via the Internet at http://www.sciencedirect.com/.

\section{References}

[1] Trovarelli, A., Fornasiero, P., Eds. Catalysis by Ceria and Related Materials, 2nd ed.; Catalytic Science Series; Imperial College Press: London, 2013; Vol. 12.

[2] Gorte, R. J. Ceria in Catalysis: From Automotive Applications to the Water Gas Shift Reaction. AICHE 2010, 56, 1126 - 1135.

[3] Vivier, L.; Duprez, D. Ceria-Based Solid Catalysts for Organic Chemistry. Chemsuschem 2010, 3, $654-678$.

[4] Chen, H.-Y.; Chang, H.-L. Development of Low Temperature ThreeWay Catalysts for Future Fuel Efficient Vehicles. Johnson Matthey Technol. Rev. 2015, 59, $64-67$.

[5] Vecchietti, J.; Bonivardi, A.; Xu, W.; Stacchiola, D.; Delgado, J. J.; Calatayud, M.; Collins, S. E. Understanding the Role of Oxygen Vacancies in the Water Gas Shift Reaction on Ceria-Supported Platinum Catalysts. ACS Catalysis 2014, 4, 2088 - 2096.

[6] Yuan, Q.; Duan, H. H.; Li, L. L.; Sun, L. D.; Zhang, Y. W.; Yan, C. H. Controlled Synthesis and Assembly of Ceria-Based Nanomaterials. J. Colloid Interface Sci. 2009, 335, 151 - 167. 
[7] Li, M.; Wu, Z.; Overbury, S. H. Surface Structure Dependence of Selective Oxidation of Ethanol on Faceted $\mathrm{CeO}_{2}$ Nanocrystals. J. Cat. 2013, $306,164-176$.

[8] Mullins, D. R.; Albrecht, P. M.; Calaza, F. C. Variations in Reactivity on Different Crystallographic Orientations of Cerium Oxide. Top. Catal. 2013, 56, $1345-1362$.

[9] Mullins, D. The Surface Chemistry of Cerium Oxide. Surf. Sci. Rep. 2015, 70, $42-85$.

[10] Mann, A. K. P.; Wu, Z. L.; Overbury, S. H. In Catalysis by Materials with Well-Defined Structures; Wu, Z., Overbury, S. H., Eds.; Elsevier, 2015; Chapter The Characterization and Structure-Dependent Catalysis of Ceria with Well-Defined Facets, pp $71-97$.

[11] Wu, Z.; Li, M.; Mullins, D. R.; Overbury, S. H. Probing the Surface Sites of $\mathrm{CeO}_{2}$ Nanocrystals with Well-Defined Surface Planes via Methanol Adsorption and Desorption. ACS Catal. 2012, 2, 2224 - 2234.

[12] Beste, A.; Mullins, D. R.; Overbury, S. H.; Harrison, R. J. Adsorption and Dissociation of Methanol on the Fully Oxidized and Partially Reduced (111) Cerium Oxide Surface: Dependence on the Configuration of the Cerium 4 f Electrons. Surface Science 2008, 602, 162 - 175.

[13] Beste, A.; Overbury, S. H. Pathways for Ethanol Dehydrogenation and Dehydration Catalyzed by Ceria (111) and (100) Surfaces. J. Phys. Chem. C 2015, 119, $2447-2455$. 
[14] Paier, J.; Penschke, C.; Sauer, J. Oxygen Defects and Surface Chemistry of Ceria: Quantum Chemical Studies Compared to Experiment. Chem. Rev. 2013, 113, $3949-3985$.

[15] Mei, D.; Deskins, N. A.; Dupuis, M.; Ge, Q. Methanol Adsorption on the Clean $\mathrm{CeO}_{2}(111)$ Surface: A Density Functional Theory Study. J. Phys. Chem. C 2007, 111, $10514-10522$.

[16] Mei, D.; Deskins, N. A.; Dupuis, M.; Ge, Q. Density Functional Theory Study of Methanol Decomposition on the $\mathrm{CeO}_{2}(110)$ Surface. J. Phys. Chem. C 2008, 112, $4257-4266$.

[17] Kropp, T.; Paier, J. Reactions of Methanol with Pristine and Defective Ceria (111) Surfaces: A Comparison of Density Functionals. J. Phys. Chem. C 2014, 118, $23690-23700$.

[18] Capdevila-Cortada, M.; García-Melchor, M.; López, N. Unraveling the Structure Sensitivity in Methanol Conversion on $\mathrm{CeO}_{2}$ : A DFT $+\mathrm{U}$ Study. J. Cat. 2015, 327, $58-64$.

[19] Kropp, T.; Paier, J.; Sauer, J. Support Effect in Oxide Catalysis: Methanol Oxidation on Vanadia/Ceria. J. Am. Chem. Soc. 2014, 136, $14616-14625$.

[20] Mei, D.; Deskins, N. A.; Dupuis, M. A Density Functional Theory Study of Formaldehyde Adsorption on Ceria. Surface Science 2007, 601, 4993 $-5001$.

[21] Teng, B.-T.; Jiang, S.-Y.; Yang, Z.-X.; Luo, M.-F.; Lan, Y.-Z. A Density 
Functional Theory Study of Formaldehyde Adsorption and Oxidation on $\mathrm{CeO}_{2}$ (111) Surface. Surface Science 2010, 604, 68 - 78.

[22] Kulkarni, D.; Wachs, I. E. Isopropanol Oxidation by Pure Metal Oxid Catalysts: Number of Active Sites and Turnover Frequencies. Appl. Catal. A: General 2002, 237, $121-137$.

[23] Mullins, D. R.; Senanayake, S. D.; Chen, T.-L. Adsorption and Reaction of $\mathrm{C}_{1}-\mathrm{C}_{3}$ Alcohols Over $\mathrm{CeO}_{\mathrm{x}}(111)$ Thin Films. J. Phys. Chem. C 2010, 114, $17112-17119$.

[24] Mullins, D. R.; Robbins, M. D.; Zhou, J. Adsorption and Reaction of Methanol on Thin-Film Cerium Oxide. Surface Science 2006, 600, 1547 $-1558$.

[25] Siokou, A.; Nix, R. M. Interaction of Methanol with Well-Defined Ceria Surfaces: Reflection/Absorption Infrared Spectroscopy, X-Ray Photoelectron Spectroscopy, and Temperature-Programmed Desorption Study. J. Phys. Chem. B 1999, 103, $6984-6997$.

[26] Ferrizz, R. M.; Wong, G. S.; Egami, T.; Vohs, J. M. Structure Sensitivity of the Reaction of Methanol on Ceria. Langmuir 2001, 17, $2464-2470$.

[27] Molinari, M.; Parker, S. C.; Sayle, D. C.; Islam, M. S. Water Adsorption and Its Effect on the Stability of Low Index Stoichiometric and Reduced Surfaces of Ceria. J. Phys. Chem. C 2012, 116, $7073-7082$.

[28] Wu, X.-P.; Gong, X.-Q.; Lu, G. Role of Oxygen Vacancies in the Surface Evolution of $\mathrm{H}$ at $\mathrm{CeO}_{2}$ (111): a Charge Modification Effect. Phys. Chem. Chem. Phys. 2015, 17, $3544-3549$. 
[29] Marrocchelli, D.; Yildiz, B. First-Principles Assessment of $\mathrm{H}_{2} \mathrm{~S}$ and $\mathrm{H}_{2} \mathrm{O}$ Reaction Mechanisms and the Subsequent Hydrogen Absorption on the $\mathrm{CeO}_{2}(111)$ Surface. J. Phys. Chem. C 2012, 116, $2411-2424$.

[30] Watkins, M. B.; Foster, A. S.; Shluger, A. L. Hydrogen Cycle on $\mathrm{CeO}_{2}$ (111) Surfaces: Density Functional Theory Calculations. J. Phys. Chem. C 2007, 111, $15337-15341$.

[31] Blochl, P. E. Projector Augmented-Wave Method. Phys. Rev. B 1994, $50,17953-17979$.

[32] Kresse, G.; Joubert, D. From Utrasoft Pseudopotentials to the Projector Augmented-Wave Method. Phys. Rev. B 1999, 59, 1758 - 1775.

[33] Kresse, G.; Hafner, J. Ab Initio Molecular Dynamics for Liquid Metals. Phys. Rev. B 1993, 47, $558-561$.

[34] Kresse, G.; Hafner, J. Ab Initio Molecular Dynamics Simulation of the Liquid-Metal-Amorphous-Semiconductor Transition in Germanium. Phys. Rev. B 1994, 49, $14251-14269$.

[35] Kresse, G.; Furthmüller, J. Efficiency of Ab Initio Total Energy Calculations for Metals and Semiconductors Using a Plane-Wave Basis Set. Comput. Mat. Sci. 1996, 6, $15-50$.

[36] Kresse, G.; Furthmüller, J. Efficient Iterative Schemes for Ab Initio Total Energy Calculations Using a Plane-Wave Basis Set. Phys. Rev. B 1996, 54, $11169-11186$. 
[37] Perdew, J. P.; Burke, K.; Ernzerhof, M. Generalized Gradient Approximation Made Simple. Phys. Rev. Lett. 1996, 77, 3865 - 3868.

[38] Dudarev, S. L.; Botton, G. A.; Savrasov, S. Y.; Humphreys, C. J.; Sutton, A. P. Electron-Energy-Loss Spectra and the Structural Stability of Nickel Oxide: An LSDA+U sudy. Phys. Rev. B 1998, 57, 1505 - 1509.

[39] Huang, M.; Fabris, S. CO Adsorption and Oxidation on Ceria Surfaces from DFT+U Calculations. J. Phys. Chem. C 2008, 112, $8643-8648$.

[40] Loschen, C.; Carrasco, J.; Neyman, K. M.; Illas, F. First-principles $\mathrm{LDA}+\mathrm{U}$ and GGA $+\mathrm{U}$ study of cerium oxides: Dependence on the effective U parameter. Phys. Rev. B 2007, 75, 035115-1-8.

[41] Calaza, F. C.; Xu, Y.; Mullins, D. R.; Overbury, S. H. Oxygen VacancyAssisted Coupling and Enolization of Acetaldehyde on $\mathrm{CeO}_{2}(111)$. $J$. Am. Chem. Soc. 2012, 134, $18034-18045$.

[42] Castleton, C. W. M.; Kullgren, J.; Hermansson, K. Tuning LDA+U for Electron Localization and Structure at Oxygen Vacancies in Ceria. $J$. Chem. Phys. 2007, 127, 244704-1-11.

[43] Fernández-Torre, D.; Kośmider, K.; Carrasco, J.; GandugliaPirovano, M. V.; Pérez, R. Insight in to the Adsorption of Water on the Clean $\mathrm{CeO}_{2}(111)$ Surface with van der Waals and Hybrid Density Functionals. J. Phys. Chem. C 2012, 116, $13584-13593$.

[44] Grimme, S.; Antony, J.; Ehrlich, S.; Krieg, H. A Consistent and Accurate ab initio Parametrization of Density Functional Dispersion Correction 
(DFT-D) for the 94 Elements H-Pu. J. Chem. Phys. 2010, 132, 154104$1-154104-19$.

[45] Jónsson, H.; Mills, G.; Jacobsen, K. W. In Classical and Quantum Dynamics in Condensed Phase Simulations; Berne, B. J., Ciccotti, G., Coker, D. F., Eds.; World Scientific: New Jersey, 1998; Chapter Nudged Elastic Band Method for Finding Minimum Energy Paths of Transitions, p 385.

[46] Henkelman, G.; Uberuaga, B. P.; Jónsson, H. A Climbing Image Nudged Elastic Band Method for Finding Saddle Points and Minimum Energy Paths. J. Chem. Phys. 2000, 113, $9901-9904$.

[47] Tang, W.; Sanville, E.; Henkelman, G. A Grid-Based Bader Analysis Algorithm Without Lattice Bias. Condens. Matter 2009, 21, 084204-1 $-7$.

[48] Sanville, E.; Kenny, S. D.; Smith, R.; Henkelman, G. An Improved GridBased Algorithm for Bader Charge Analysis. J. Comp. Chem. 2007, 28, $899-908$.

[49] Henkelman, G.; Arnaldsson, A.; Jónsson, H. A Fast and Robust Algorithm for Bader Decomposition of Charge Density. Comput. Mater. Sci. 2006, 36, $254-360$.

[50] Zhou, J.; Mullins, D. R. Adsorption and Reaction of Formaldehyde on Thin-Film Cerium Oxide. Surf. Sci. 2006, 600, 1540 - 1546.

[51] Redhead, P. A. Thermal Desorption of Gases. Vacuum 1962, 12, 203 211. 
[52] Fernández-Torre, D.; Carrasco, J.; Ganduglia-Pirovano, M. V.; Pérez, R. Hydrogen Activation, Diffusion, and Clustering on $\mathrm{CeO}_{2}(111)$ : A DFT+U Study. J. Chem. Phys. 2014, 141, 014703-1-014703-9. 
Graphical Abstract

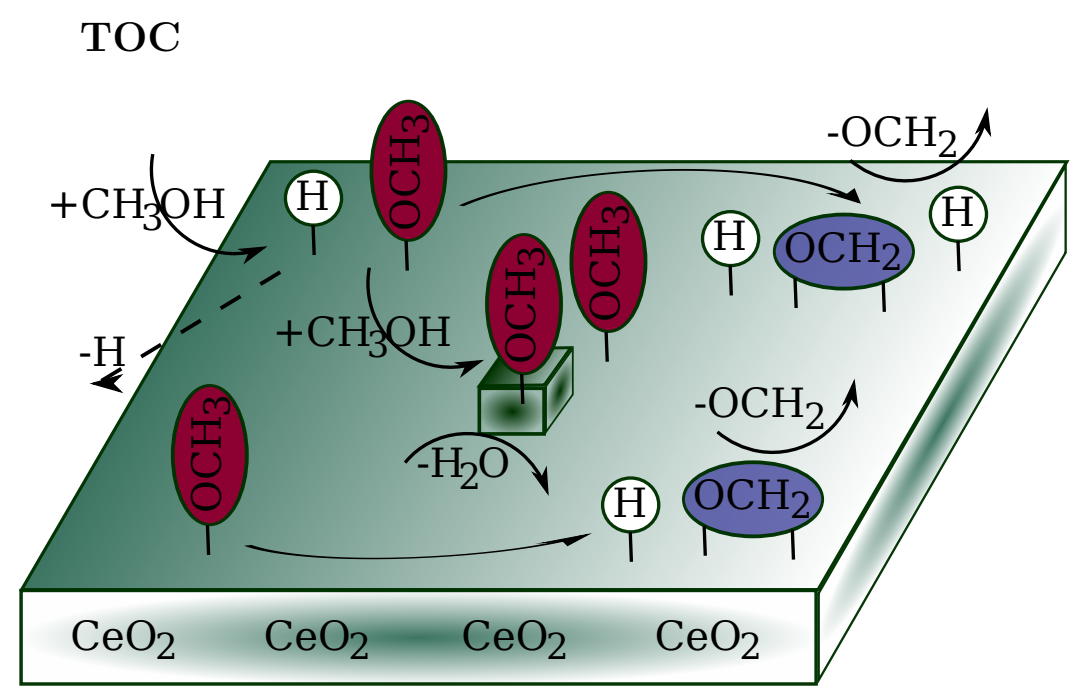

